\title{
A Avaliação Externa de Escolas na perspetiva dos Coordenadores de Autoavaliação
}

\section{School External Evaluation in the perspective of the self-evaluation coordinators}

\author{
Natália Costa, Joana Sousa \\ Universidade do Minho, Portugal
}

\begin{abstract}
Resumen
Em Portugal a Avaliação Externa de Escolas é consagrada pela Lei $\mathrm{n}^{\mathrm{o}} 31 / 2002$, de 20 de fevereiro, tendo sido implementada, a partir de 2005/06, pela Inspeção Geral de Educação e Ciência. Este modelo constitui-se como um identificador de boas de práticas organizativas, procedimentais e pedagógicas das instituições educativas. Pretende criar de forma duradoura e sistemática uma cultura de qualidade e melhoria através da criação de níveis de eficiência e eficácia que permitam a contextualização e interpretação dos resultados com vista à credibilização e ao sucesso do sistema educativo. A Avaliação Externa de Escolas (AEE) surge enquadrada em políticas educativas nacionais e internacionais que promovem a legitimação do conceito de avaliação colocando a accountability, a qualidade, a melhoria, a eficácia, a eficiência e os resultados como objetivo primordial na avaliação de instituições. Neste contexto surge a questão: Quais as consequências da AEE na perspetiva dos Coordenadores das equipas de autoavaliação de escolas da região norte de Portugal?. Para a compreensão do conceito de avaliação recorreu-se à análise de diferentes definições, sendo que a análise de conteúdo das entrevistas dos Coordenadores da equipa de autoavaliação $(n=2)$ de agrupamentos de escola da região norte de Portugal, baseia-se na perspetiva de avaliação de Nevo (2007) que considera que a avaliação compreende cinco funções: tomada de decisão, melhoria, accountability, profissionalismo e certificação. Através da análise das entrevistas verifica-se uma relação entre a AEE e as funções apontadas por Nevo (2007), na medida em que é um processo que legitima a tomada de decisões das lideranças intermédias das instituições educativas na busca da melhoria, responsabilizando os diversos atores e certificando aos olhos da sociedade as práticas organizativas, pedagógicas e curriculares desenvolvidas pelas escolas. Os dados do estudo apontam para a importância do contexto no desenvolvimento da equipa de autoavaliação, bem como no reconhecimento e no trabalho desenvolvido pelas equipas.

Palavras-chave: avaliação externa de escolas, tomada de decisão, accountability, melhoria, certificação
\end{abstract}

\begin{abstract}
In Portugal, Schools External Evaluation is established in Law no. 31/2002 of 20th February, having been implemented from 2005/06, by the General Inspection of Education and Science. This model constitutes itself as an identifier of good organizational, procedural and pedagogical practices of the educational institutions. It
\end{abstract}

intends to create a lasting and systematic culture of quality and improvement by creating levels of efficiency and efficacy that enable the contextualization and interpretation of the results to accomplish credibility and success in the education system. Schools External Evaluation (SEE) comes framed in national and international educational policies that support the legitimacy of the concept of evaluation placing accountability, quality, improvement, efficacy, efficiency and results as main objectives of the evaluation of institutions. In this context a question arises: What are the consequences of SEE in the perspective of school self-evaluation coordinators of Portugal's northern region?. To understand the concept of evaluation we resorted to the analysis of different definitions and the content analysis of the interviews of self-evaluation coordinators of school clusters $(\mathrm{n}=2)$ in the northern region of Portugal, based on the evaluation prospective of Nevo (2007) that considers it comprehends five functions: decision making, improvement, accountability, professionalism and certification. Through the analysis of the interviews we found a correlation between SEE and the functions acknowledged by Nevo (2007), since it is a process that legitimizes the decision-making of the intermediate leaders of educational institutions looking for improvement and holds responsible various actors certifying, at the eyes of society, organizational, pedagogical and curricular practices carried out by schools. The data point to the recognition of the importance of the educational context and the work done by the self-evaluation coordinators. This communication it's inserted on an investigation project called "School External Evaluation on the non-higher education" (FCT PTDC/CPE-CED/116674/2010) led by Minho's University.

Keywords: school external evaluation, decision making, accountability, improvement, certification.

\section{Introdução}

Nos últimos anos, a Avaliação tornou-se uma prática debatida publicamente, baseada em diferentes conceitos que fomentaram a ideia de um Estado-avaliador e regulador. A necessidade de definição de objetivos e a mensuração evoluíram a par de uma nova gestão pública, baseada numa economia globalizada. Assim, a Avaliação focaliza-se na performatividade e na accountability, seguindo ideais neoliberais (Carpenter, Diem \& Young, 2014). Na educação, esta visão promoveu a ideia de que as escolas são serviços públicos que devem prestar contas ao público e às organizações nacionais e

Esta comunicação insere-se no projeto de investigação de Avaliação Externa de Escolas no Ensino Não Superior (FCT PTDC/CPE-CED/116674/2010) coordenado pela Universidade do Minho.

Natália Costa, natáliacosta.costa6@gmail.com

Joana Sousa,joanarfsousa@gmail.com. Bolseira de investigação científica em Ciências da Educação, especialização em Desenvolvimento Curricular pela Fundação para a Ciência e a Tecnologia (FCT - SFRH/BD/93389/2013). 
transnacionais. A avaliação é reconhecida como um instrumento fundamental na reforma das instituições que procuram responder ao mercado, tendo na sua retaguarda o Estado com o objetivo de "produzir mais qualidade, pertinência e sentido público ao conjunto das atividades académicas, científicas, técnicas e administrativas, bem como, às relações sociais, interinstitucionais e intrainstitucionais." (Sobrinho, 2003, p. 10). Em resposta, as escolas alteraram as suas práticas administrativas, adaptando-se à gestão de mercado "focusing mainly on the future demand for labor, social efficiency, market competition, and league tables of performance between schools, systems of educational testing that are tied to the criteria for market competition." (Saari, Salmela \& Vilkkilä, 2014, p. 184). Na sua diversidade de objeto e na sua problematização, a avaliação institucional tem obedecido quase exclusivamente a uma função de regulação, com peso do lado sumativo da avaliação, como se constata em diversos relatórios internacionais (OECD, 2013; Eurydice, 2015).

De acordo com o quadro teórico que a sustenta a Avaliação é, essencialmente, formativa e sumativa (Scriven, 1967; Nevo, 2007), sendo encarada como duas faces de uma mesma moeda, uma que proporciona a melhoria, o desenvolvimento e a aprendizagem (Scheerens, 2003; Pacheco, 2010), enquanto a outra promove a prestação de contas (Afonso, 2009; Fullan, Rincon-Gallardo \& Hargreaves, 2015), numa lógica de comparação de resultados e competitividade (Ozga \& Grek, 2012) recorrendo a conceitos-chave, tais como qualidade, eficácia e eficiência. Mesmo que em determinados modelos e práticas haja a tendência para a focalização de uma delas, a avaliação pressupõe a melhoria escolar, contribuindo de forma reflexiva para a partilha contínua interna (Bolívar 2003, 2012).

Na perspetiva de Nevo (2007) a Avaliação compreende cinco funções: tomada de decisão, melhoria, accountability, profissionalismo e certificação. Estas cinco funções estão relacionadas com as necessidades, propósitos e níveis do sistema educativo, encontrando-se presente em todo o domínio educacional. Reflexo disso é a abrangência da função de tomada de decisão que envolve alunos, professores, pais e administradores. Numa lógica de inovação e modernização surge a função de melhoria que promove a aprendizagem dos alunos, a melhoria das competências dos professores, assim como a atualização e o desenvolvimento constante dos materiais educativos. A função de accountability torna-se incompleta quando usada apenas na interpretação de resultados, quer de alunos, quer da Avaliação externa, sendo completa se recorrer ao diálogo contínuo entre professores, escolas e Avaliação externa. Ao nível do profissionalismo pretende-se que as necessidades profissionais dos docentes tenham um impacto positivo no ensino, através da promoção de práticas de autoavaliação contínuas que favoreçam as opções curriculares de forma intencional. Desta forma, fomentar-se-á a participação ativa dos professores na AEE, tornando-se parte integrante da vida dos docentes. A Avaliação é um recurso amplamente utilizado para a certificação das instituições educativas, das administrações escolares, dos programas educativos e dos professores. Esta esfera avaliativa torna a avaliação legítima e justifica-a perante a sociedade sendo uma forma de acreditação ou reconhecimento formal.

\section{Avaliação Externa de Escolas}

A publicação da Lei n³1/2002, de 20 de dezembro, aprova o sistema de avaliação nos estabelecimentos de educação pré-escolar e dos ensinos básico e secundário, mediante a implementação de um modelo de AEE, que se encontra enquadrado em processos de regulação transnacional e supranacional e em políticas que promovem a prestação de contas e a responsabilização (Afonso, 2009), associando-se à autonomia das escolas, ao desenvolvimento profissional docente e à eficácia e melhoria das escolas.

A avaliação de escolas é uma atividade de legitimação legal, de acordo com a Lei de Bases do Sistema Educativo, da responsabilidade da Inspeção-geral da Educação e Ciência (IGEC), assumindo-a "como um contributo para o desenvolvimento das escolas" (IGE, 2011, p. 51), competindo ao Conselho Nacional de Educação a sua avaliação. Sendo apresentado como um instrumento formativo de avaliação da qualidade da escola, o modelo de AEE tem em conta as primeiras experiências deste organismo central ligadas à avaliação institucional, seguindo as orientações europeias, definidas pela European Foundation for Quality Management (EFQM), e adotando os princípios do modelo escocês How Good is Our School.

Em Portugal o sistema de AEE compreende dois ciclos. O primeiro ciclo, iniciado em 2006 e terminado em 2011, incidiu nos domínios dos resultados, prestação do serviço educativo, organização e gestão escolar, liderança e capacidade de autorregulação e melhoria da escola/agrupamento. O segundo ciclo, iniciado em 2012, incidiu no domínio dos resultados, prestação do serviço educativo e liderança e gestão. Cada um destes domínios é fundamentado por fatores e indicadores. Como resultado da AEE surge uma classificação que presentemente é a seguinte: Excelente, Muito Bom, Bom, Suficiente e Insuficiente.

Ao longo da implementação da AEE é possível verificar que a mesma está intrinsecamente relacionada com as políticas educativas de cada Estado (Pacheco, 2014) sendo, claramente, um domínio social influenciado por conceitos geradores de mudanças ao nível das práticas organizacionais, curriculares e pedagógicas (Rodrigues, Queirós, Sousa, Costa, 2014). Tal como defende Ball (1997), no ciclo de construção das políticas, o contexto de influência é um dos vetores mais determinantes na regulação que é exercida pelos organismos transnacionais, que são o berço das políticas de partilha de conhecimento e das políticas de accountability.

\section{Metodologia}

Com o objetivo de responder à questão de investigação optamos por uma abordagem qualitativa de cariz interpretativo "que nos permita estabelecer uma compreensão mais esclarecedora do nosso objeto de estudo." (Bodgan \& Biklen, 1994, p.49). Utilizamos 
como técnica de recolha de dados a análise de conteúdo (Esteves, 2006) de entrevistas semiestruturadas $(n=2)$ a Coordenadores da equipa de autoavaliação de agrupamentos de escolas da zona Norte de Portugal. Para analisar os dados (E1, E2), utilizamos a análise de conteúdo recorrendo a unidades de registo semânticas (URS) (Esteves, 2006) que estejam relacionadas com as funções enumeradas por Nevo (2007): tomada de decisão, melhoria, accountability, profissionalismo e certificação.

Para efetuar a recolha de dados recorremos a um quadro onde estão registadas as funções mencionadas por Nevo (2007) e as URS. Desta forma conseguimos estabelecer relação entre o discurso dos Coordenadores da equipa de autoavaliação, as funções de avaliação (Nevo, 2007) e as consequências da AEE. Com o objetivo de "isolar os sentidos diversos presentes no que foi dito" (Esteves, 2006, p.114).

No que diz respeito às questões éticas foram utilizados diversos princípios, nomeadamente ao nível do consentimento informado e da garantia da confidencialidade e anonimato das instituições e dos respondentes (Lima, 2006; Quivy \& Campenhoudt, 2005; Tuckman, 2000).

\section{Resultados e Discussão}

A análise de conteúdo realizada tem como principal objetivo compreender as perspetivas dos Coordenadores de equipa de autoavaliação entrevistados relativamente às consequências da AEE enquadradas na lógica de avaliação defendida por Nevo (2007). De modo a escrutinar os dados que emergem da análise de conteúdo propomos fazê-lo com base nas funções apresentadas por Nevo (2007). Apesar de apresentarmos duas entrevistas de Coordenadores de equipa de autoavaliação em contextos diferentes da zona Norte de Portugal, ambas remetem-nos para experiências semelhantes, embora a prática de autoavaliação, numa das escolas, seja apoiada por uma equipa externa, não existindo, por isso, uma equipa formalmente constituída.

Relativamente à função de tomada de decisão, através da análise das entrevistas, observa-se que as equipas de autoavaliação detêm responsabilidades limitadas, uma vez que, por exemplo, em relação à redação do plano de melhoria o Coordenador (E1) refere que "Isso é realizado pelos departamentos e, naturalmente, a direção.”. No caso do E2 "a tal equipa (...) também não tem tido nas suas competências elaborar qualquer plano de melhoria", sendo que apesar da equipa de autoavaliação identificar os problemas da escola e poder "eventualmente (...) propor uma ou outra solução, mas fica-se por aí." (E1), sendo que "a grande credibilidade do trabalho feito pela equipa advém de não comentar, não avaliar (...) sendo as conclusões tiradas pelos órgãos pedagógicos da escola" (E2). Em ambos os casos (E1 e E2), os Diretores têm um papel preponderante, não só na realização do plano de melhoria, como também na monitorização do mesmo. Todas estas questões devem ser analisadas à luz do contexto do processo de AEE, que na perspetiva do E1 condiciona toda a ação do Diretor: “(...) penso que sobretudo os diretores devem sentir mais pressão por parte da tutela para melhorarem os resultados escolares!". Em suma, os dados das entrevistas deixam transparecer o efeito exógeno da AEE (Ehren \& Visscher, 2006), visto que o modelo leva a uma uniformização forçada em que a "administração pode prescrever e prescreve, uniformiza e centraliza", refletindo-se essa pressão no relatório de AEE, tal como é referido na E2: "A observação menos favorável que tivemos nesse âmbito foi precisamente não termos formalmente essa tal equipa", demonstrando que apesar de a legislação apontar apenas para a obrigatoriedade de práticas de autoavaliação, a não existência de uma equipa acaba por ser penalizadora para as escolas.

Uma das questões apontadas pelos Coordenadores de equipa de autoavaliação (E1 e E2) reflete a função de accountability, através da predominância do domínio dos resultados face aos restantes avaliados pela AEE: “( ...) somos avaliados pelos resultados." (E2), contribuindo, deste modo, para uma valorização dos resultados académicos (Sousa, Costa, Rodrigues, Queirós, Lamela, Seabra \& Morgado, 2015), sobretudo ao nível da disciplina de Português e Matemática visto serem consideradas áreas prioritárias, dado o peso das classificações dos exames destas disciplinas nos resultados da escola. Ao nível pedagógico e para o E1 este olhar centrado nos resultados e nestas duas disciplinas não é benéfico, no entanto, por se demonstrarem ser preponderantes nos resultados de AEE, a escola procura orientar os seus recursos para controlar a sustentação de práticas que resultem na melhoria dos resultados académicos, produzindo, tendencialmente, o efeito perverso de distração dos atores educativos e da comunidade envolvente para o curto prazo, resultando num efeito de miopia (Ehren \& Visscher, 2006), tal como é levantado por um dos Coordenadores (E1): “(...) mas isto é um bocado perverso, não é? Porque as escolas começam a trabalhar para a avaliação externa e não para os alunos!". Um exemplo disto mesmo é o facto de a articulação do $1^{\circ}$ para o $2^{\circ}$ ciclo ser uma área de melhoria e de preocupação por parte da escola, ao contrário da articulação entre o pré-escolar e o $1^{\circ}$ ciclo, "porque do primeiro para o segundo ciclo já se trabalha com resultados, ao contrário do pré-escolar para o primeiro ciclo..." (E1). Esta propensão para a tomada de decisão pragmática, centrada nos resultados da escola, potencia a contínua segmentação e particularização da escola, que se reflete no aumento da burocracia fomentada no conceito da necessidade em se impor perante os resultados. Para contrariar o excesso de burocracia surgem as novas tecnologias que permitem reduzir o tempo disponibilizado no registo e sistematização de evidências, tal como é referido em ambas as entrevistas (E1 e E2). No entanto, para combater esta tendência para o curto prazo não existem dados nas entrevistas que permitam indicar que algo está a ser colocado em prática, aliás, é mesmo afirmado pelo E1 que "Se o objetivo é trabalharmos todos para os resultados, eles estão a trabalhar bem", deixando para reflexão a questão de qual é que é o objetivo final do processo de AEE. Tendo em conta as recomendações da OCDE (2013) a AEE deve compreender a realidade da escola, destacando a 
importância do ensino e da aprendizagem, para além dos resultados dos alunos, salientando o papel central do Estado na clarificação dos propósitos da AEE.

Quanto à função de melhoria, para os Coordenadores de equipa de autoavaliação (E1 e E2) a AEE não provoca uma melhoria evidente: “(...) não foi o facto de uma avaliação externa que fez com que fossem criados mecanismos de autoavaliação" (E2). Na E1 levanta-se uma questão pertinente sobre a ligação entre a autoavaliação e a AEE quando é afirmado que "O modelo de autoavaliação está construído de maneira a responder ao modelo da avaliação externa.”. Dado que este modelo tem como objetivo "incrementar a responsabilização a todos os níveis, validando as práticas de autoavaliação das escolas" (IGEC, 2012) podemos afirmar que existe uma tendência para a autoavaliação estar conforme a AEE para que seja validada pela mesma. No entanto, esta visão da autoavaliação pode tornar-se reducionista quando para a IGEC o processo de AEE pretende "contribuir para o desenvolvimento das escolas" (IGE, 2011). Através da análise das entrevistas, verifica-se que a autoavaliação não está a ser preponderante para o aumento da reflexão nas escolas. Esta situação demonstra o enfoque da AEE nos resultados em detrimento da melhoria pedagógica e curricular.

Ao nível do profissionalismo, a E1 remete para as diferenças entre as equipas de AEE entre o $1^{\circ}$ e o $2^{\circ}$ ciclo de avaliação, ao contrário da E2, podendo-se relacionar com as diferentes formas de apropriação da AEE por parte das escolas. Salienta-se que na escola do E2 já existiam práticas de autoavaliação consolidadas e por isso, o $1^{\mathrm{o}}$ ciclo de AEE foi encarado como uma oportunidade de melhoria. $\mathrm{Na}$ perspetiva do E1, o $1^{\mathrm{o}}$ ciclo de AEE, é visto como uma ameaça que promoveu junto dos atores escolares a construção e o enraizamento da identidade da escola provocando a adoção do referencial externo para a autoavaliação. Cruzando a análise das entrevistas, os dados da escola e as características da Inspeção, podemos dizer que no $1^{\circ}$ ciclo de AEE nestas escolas a abordagem da Inspeção foi tendencialmente diretiva (Pacheco, Seabra, Morgado, 2014), ao passo que no $2^{\circ}$ ciclo de AEE as escolas (E1 e E2) perspetivaram este processo de uma forma mais otimista, facto que é corroborado por Rodrigues e Moreira (2015).

Os resultados da AEE são vistos como pouco legítimos uma vez que "ao texto em si, não temos muito a acrescentar, agora, a conversão do texto no resultado qualitativo é que nos levantou dúvidas..." (E1), revelando que a certificação é vista como uma confirmação das fragilidades da escola e das boas práticas exercidas, sobretudo na perspetiva da comunidade: "Esta avaliação veio, quando muito, funcionar como um fator de orgulho para os pais." (E2). O discurso dos Coordenadores demonstra o caracter formativo diminuto da AEE, continuando a existir necessidades sistémicas: “(...) não acho que o facto de termos uma equipa dê maior credibilidade àquilo que essa equipa faz."(E2). Para o Coordenador da equipa de autoavaliação (E1) o contexto educativo é um fator que deveria ser tido em conta com maior vigor no processo de
AEE, nomeadamente as condições socioeconómicas das famílias que no geral não revelam grande preocupação em que os seus filhos tenham sucesso e por isso não contribuem de modo coerente para a evolução do seu desempenho escolar. Do ponto de vista do Coordenador (E1) a "formatação" do processo de AEE não permite que estes dados contextuais sejam ponderados na avaliação que é feita à escola, havendo um hiato entre os resultados da AEE e o que é vivido nas escolas: "Não senti que a avaliação externa estivesse longe da verdade ou do que nós somos agora (...)." (E2). Sobre o papel da AEE o E1 menciona uma questão pertinente no segundo processo de AEE, quando afirma que “(...) esta última equipa que cá esteve até foi positiva, porque ouviu de facto as pessoas, etc.". Assim, revela-se importante a forma como a equipa de AEE é encarada, tendendo a ser mais valorizada quando é vista como um processo formativo em detrimento de sumativo. Na perspetiva do Coordenador (E1), o papel da autoavaliação na escola "é alertar para algo que seja necessário melhorar", apontando para uma autoavaliação centrada na solução de problemas imediatos e pouco ou nada focada na melhoria da escola a longo prazo. Acerca das mudanças ao nível organizacional existe um efeito de ossificação (Ehren \& Visscher, 2006), na medida em que há uma procura da escola em corresponder às dinâmicas pontuais indicadas pela AEE.

\section{Referencias}

Afonso, A. (2009). Nem tudo o que conta em educação é mensurável ou comparável. Crítica à accountability baseada em testes estandardizados e rankings escolares. Revista Lusófona de Educação, 13, 13-29. Retrieved from http://revistas.ulusofona.pt/index.php/rleducacao/artic le/view/545/447

Ball, S. (1997). Education reform. A critical and post-structural approach ( $2^{\mathrm{a}}$ ed.). Oxford: Oxford Open University Press.

Bogdan, R. \& Biklen, S. (1999). Investigação qualitativa em educação. Uma introdução à teoria e aos métodos. Porto: Porto Editora.

Bolívar, A. (2003). Como melhorar as Escolas estratégias e dinâmicas de melhoria das práticas educativas. Porto: Edições ASA.

Bolívar, A. (2012). Melhorar os Processos e os Resultados Educativos: $O$ que nos ensina a investigação. Vila Nova de Gaia: Fundação Manuel Leão.

Carpenter, B, Diem, S. \& Young, M. (2014). The influence of values and policy vocabularies on understandings of leadership effectiveness. International Journal of Qualitative Studies in Education, 27 (9), 1110-1133. DOI: 10.1080/09518398.2014.916008

Ehren, M. \& Visscher, A. (2006). Towards a theory of schools inspection, 54 (1), 51-72.

Ehren, M. \& Visscher, A. (2006). Towards a theory on the impact of schools inspections. British Journal of Educational Studies. 54 (1), 51-72. ISSN 0007-1005 
Esteves, M. (2006). Análise de Conteúdo. In J. Lima \& J. Pacheco (Org.), Fazer investigação. Contributos para a elaboração de dissertações e teses (pp. 105-126). Porto: Porto Editora.

European Commission/EACEA/Eurydice (2015). Assuring Quality in Education: Policies and Approaches to School Evaluation in Europe. Eurydice Report. Luxembourg: Publications Office of the European Union. Retrieved from http://eacea.ec.europa.eu/education/eurydice/documen ts/thematic_reports/178EN.pdf

Fullan, M., Rincon-Gallardo, S. \& Hargreaves, A. (2015). Professional Capital as Accountability. Education Policy Analysis Archives, 23 (15), 1-22.

IGE (2001). Plano de atividades. Instrumentos de gestão. Lisboa: IGE.

IGE (2011). IGE. Plano de atividades. Instrumentos de gestão. Lisboa: IGE.

IGEC (2012). Quadro de referência para a avaliação das escolas. Lisboa: IGE.

Lima J. (2006). Ética na investigação. In J. Lima \& J. Pacheco (Org.), Fazer investigação. Contributos para a elaboração de dissertações e teses (pp. 127-159). Porto: Porto Editora.

Nevo, D. (2007). Evaluation in Education. In I. F. Shaw, J.C. Green \& M. M. Mark (Eds.), Handbook of evaluation. Policies, programs and practices (pp.440-460). London: Sage Publications.

OCDE (2012). Reviews of evaluation and assessment in education: Portugal 2012. Paris: OECD. Retrieved from http://www.oecd.org/edu/school/50077677.pdf.

OCDE (2013). Education at a Glance 2013. OECD indicators. Retrieved form www.oecd.org/edu/eag2013\%20(eng)--FINAL\%2020 \%20June\%202013.pdf

OECD (2013). Synergies for Better Learning: An international perspective on evaluation and assessment. Paris: OECD. http://dx.doi.org/10.1787/9789264190658-en

Ozga, J. \& Grek, S. (2012). Governing Through Learning: school self-evaluation as a knowledge-based regulatory tool. Reserches Sociologiques et Anthropologies, 43 (2), 83-103.

Pacheco, J. (2010). Avaliação Externa das Escolas: Teorias e Modelos, Seminário Avaliação Externas das Escolas: Modelos, Práticas e Impacto. Braga: Universidade do Minho.

Pacheco, J. (Org.) (2014). Avaliação Externa de Escolas: Quadro Teórico/Conceptual. Porto: Porto Editora.

Pacheco, J., Seabra, F. \& Morgado, J. (2014). Avaliação externa. Para a referencialização de um quadro teórico sobre o impacto e efeitos nas escolas do ensino não superior. In J. Pacheco (Org.), Avaliação externa de escolas: quadro teórico/conceptual. Porto: Porto Editora.

Quivy, R., \& Campenhoudt, L. (2008). Manual de investigação em Ciências Sociais. Lisboa: Gradiva.

Rodrigues, E., Queirós, H., Sousa, J., \& Costa, N. (2014). Avaliação Externa de Escolas: do referencial aos estudos empíricos. In J. Pacheco (Org.), Avaliação Externa de Escolas: Quadro Teórico/Conceptual (pp. 91-118). Porto: Porto Editora.
Rodrigues, P., Moreira, J. (2015). O que dizem os Diretores das Escolas. Comunicação apresentada no Seminário do Conselho Nacional de Educação Avaliação Externa das escolas. Coimbra, Portugal.

Saari, A., Salmela, S., \& Vilkkilä, J. (2014). Governing Autonomy. In W. Pinar (Ed.), International Handbook of Curriculum Research (2 ${ }^{\mathrm{a}}$ Ed.) (pp.183-200). New York: Taylor \& Francis.

Scheerens, J. (2003). Melhorar a Eficácia das Escolas. Porto: Edições ASA.

Scriven, M. (1967). The methodology of evaluation. In R. Tyler, R. Gagné, \& M. Scriven (Eds.), Perspectives of curriculum evaluation, 39-83. Chicago: Rand McNally.

Sobrinho, J. (2003). Avaliação: Políticas Educacionais e Reformas da Educação Superior. São Paulo: Cortez.

Sousa, J., Costa, N., Rodrigues, E., Queirós, H., Lamela, C., Seabra, F. \& Morgado, J. (2015). Avaliação externa e seus efeitos: A perspetiva dos atores escolares. In C. Barreira (Org.), Estudos sobre avaliação externa de escolas. Porto: Porto Editora.

Tuckman, B. (2002). Manual de investigação em educação: Como conceber e realizar o processo de investigação em educação. Lisboa: Fundação Calouste Gulbenkian. 